\title{
User Persona of Mother of Preterm Neonate
}

\section{Ganesh Bhutkar*, Aditya Dongre*, Shahaji Deshmukh ${ }^{\dagger}$, Lene Nielsen ${ }^{*}$ and Jaydeep Joshi*}

\author{
*Centre of Excellence in HCI, Vishwakarma Institute of Technology, \\ Pune, India \\ ${ }^{\dagger}$ Bharati Hospital, Bharati Vidyapeeth Deemed University, Pune, India \\ ${ }^{*}$ Business IT Department, IT University of Copenhagen, Denmark \\ ganesh.bhutkar@vit.edu
}

\begin{abstract}
This research paper presents a user persona of Indian mothers of preterm neonates. Many of these preterm neonates require hospitalization in Neonatal Intensive Care Units (NICUs), leading to mental stress for mothers and their families. The mother's persona is proposed based on hypothesis, user interviews and data analysis. The participant mothers of preterm neonates are graduates and homemakers from semiurban areas around Pune, India. These mothers prefer non-vegetarian diet, they visit a pediatrician more frequently and presently, they do not use any mobile healthcare app or YouTube videos for information about neonatal care. A mobile app will be developed for these mothers in future with due consideration to their user persona.
\end{abstract}

\section{Keywords}

User Persona $\cdot$ Mother $\cdot$ Preterm Neonate $\cdot$ Neonatal ICU $\cdot$ Mother's Persona

How to cite this book chapter:

Bhutkar, G., Dongre, A., Deshmukh, S., Nielsen, L. and Joshi, J. 2020. User Persona of Mother of Preterm Neonate. In: Loizides, F., Winckler, M., Chatterjee, U., Abdelnour-Nocera, J. and Parmaxi, A. (eds.) Human Computer Interaction and Emerging Technologies: Adjunct Proceedings from the INTERACT 2019 Workshops. Pp. 151-156. Cardiff: Cardiff University Press. DOI: https://doi.org/10.18573/ book3.s. License: CC-BY 4.0. 


\section{Introduction}

India is the second-most populated country in the world, with about 1365 million people [16], including about 653 million women [4]. Its population is growing, recently with a crude birth rate of 18.6 births per thousand population [2]. The effective population growth is about $1.1 \%$ per year, adding more than 15 million people to the Indian population [3]. Such high population growth results into national issues like unemployment, increased poverty level, unequal distribution of income, over-strained infrastructure, over-stretched health and educational services [12]. The challenging environment has led to deteriorating health conditions of women inflicting their pregnancies. It also results in too early, too late or too frequent pregnancies, creating complications at the time of childbirth. There is a need to use Information and Communication Technology (ICT) for advising and guiding these mothers on their health condition, along with growth tracking of their neonates, who are infants with an age of upto four weeks.

For usefulness of ICT to women in challenging healthcare conditions, the development process needs to take into account the work conditions of both the clinical staff and the mothers. The developers or researchers working with ICT do not have enough exposure to work environments such as Intensive Care Units (ICU). It generates a vital gap in the understanding of mothers of preterm neonates and their hospitalization in Neonatal Intensive Care Units (NICU). Lack of information may include understanding about mothers' requirements, preferences, day-to-day activities and the stress generated during hospitalization. This paper presents a case that aims at reducing the gap of knowledge through fieldwork in NICU and a proposed mother's persona.

\section{Related Work}

In this section, we present a few papers that are helpful in the understanding of the work environment in NICU, the psychology of parents of preterm neonates and the use of personas in health-related ICT. The papers have created a foundation for forming a hypothesis; especially related to the healthcare domain.

In an Italian study, Bouwstra et al. [1] examine the parent-to-neonate bonding experience in NICU. This research paper reveals that the parents of the hospitalized neonate are insecure about whether their neonate responds differently to them than to the medical staff. The mother is always eager to meet her neonate, especially for breastfeeding and may not be able to meet the neonate frequently throughout the day, which has been depicted in the hypothesis. The Indian research paper by Patil et al. reveals that mothers of a preterm neonate experience significant psychological distress, with elevated anxiety [12]. This study also points out the emotional problems of the mothers and their need for support. Thus, these mothers face an increased mental stress, which has been highlighted in the hypothesis. 
A study from the USA, by Heidari et al. [6] states that due to a busy occupational schedule most of the fathers find it challenging to attend to the mothers all the time during hospitalization as well as follow-up hospital visits. Thus, most of the mothers are accompanied by their mothers or relatives. This is similar to our case and is reflected in the hypothesis. Finally, a study conducted in South Africa by Mburu et al. [7] aims to use technology to support mothers of a preterm infants. It shows that the mothers get to know the neonatal status information via text message, over the phone or through digital video. Similarly, Indian mothers get a lot of neonatal information over a phone call or via text message, which is reflected in the hypothesis.

Personas [11] is a popular UX method to understand the involved users. e.g. healthcare ICT products. As mentioned by van Velsen et al. [13], it has the potential to be a useful tool for designing usable eHealth services. Looking at the field, most personas are related to self-monitoring and tracking such as a public website with cancer related information [5], a handheld device to monitor chronic heart failure [15] or wellbeing [14].

\section{Field Work}

The research has been conducted in two local hospitals located in Pune, India. The larger hospital is housed in huge multi-story buildings with a capacity of more than 500 beds. Multispecialty departments have various units like Emergency, ICU, Burn centre and NICU. Preterm neonates who need intensive medical attention are often admitted into a special area of the hospital called the Neonatal Intensive Care Unit (NICU). This unit combines advanced technology and trained healthcare professionals to provide specialized care for the tiniest patients. It has continuing care areas for neonates who have health issues and need skilled nursing care. Such NICUs are categorized in different levels [8]. NICU at Level I looks after neonates who need more care than healthy neonates and require regular monitoring assistance. NICU at Level II provides special care where nurses are assigned to 3-4 neonates for constant attention and care. NICU at Level III take care of very sick neonates providing prolonged lung ventilation-support. A neonatologist typically leads NICU and staffed with intensivists, nurses, therapists and dietitians. Neonatologists are pediatricians with additional training in the care of sick and preterm neonates [8-9].

The research in NICU has started with the design of hypothesis about mothers of preterm neonates mainly focused on their personal traits, the medical context and mobile usage in practice. A questionnaire, designed on the basis of the hypothesis, has been used for interviews of selected mothers. To facilitate the active participation of mothers, the questions for the interviews are also provided in the local regional language Marathi along with English. The field work started with interviews of physicians and nurses working with NICUs while forming the hypothesis. The main participants of these interviews have 
been 19 mothers of preterm neonates in the next phase. In all these interviews, the aim has been to get the details about demographic information, sources of information for mothers, their preferences, related facts, challenges and issues in the work environment of NICUs. The participating mothers of preterm neonates has been in the age group of 18-32 years. Most of these mothers are graduates from semi-urban areas around Pune. They were married between the age of 18 to 25 years and are housewives/home-makers. Most neonates are their first child.

The interviews have been conducted three days over a fortnight. Each interview lasted for 15-20 minutes. The research team had limited medical knowledge despite putting sufficient effort into an understanding of the neonatal context. The all-male team in Pune has faced difficulties during the initial interviewing process of mothers due to gender incompatibility and therefore, a female intern from one of the hospitals is included as an interviewer of the mothers. This intern has been there to help in sharing expectations, priorities and experiences of these mothers.

\section{Proposed User Persona}

In this qualitative research, the user is the mother of a preterm neonate. A related user persona of a mother is proposed to analyze the maternal experience in NICUs. This study aims to understand a typical Indian mother, her experience and challenges in NICU. The process of creating the neonate mother personas started with formation of a hypothesis [10]. The hypothesis is mainly focused on their personality, medical traits and active mobile usage. The assumptions include demographic information such as age, education, marriage age, area and occupation. The health and medical science traits include personal Body Mass Index (BMI), diet, medication schedule, knowledge of medical terms/equipment, mental/physical stress and others. The mobile usage section includes factors such as internet access, use of messenger app, local language support, m-Health app usage, social media groups and so on.

After proposing hypothesis, a questionnaire has been prepared for conduction of interviews of selected mothers in hospitals. This questionnaire is prepared even in Marathithe local language to facilitate active participation of mothers in interviews. It has more than 30 objective questions with upto 4 options. More than one-third questions are of YES/NO type. Using this questionnaire, interviews of mothers of preterm neonates have been conducted in NICUs. The interviews of main participant mothers have been conducted to get the details about demographic information, sources of information for mothers, their preferences, related facts and challenges in work environment of NICUs. The collected interview data has been analyzed to get vital findings/insights, capturing mother's experience in NICU. The related detailed user persona and interesting observations made on the basis of hypothesis are presented in next section. 


\section{The Mother Persona}

The results of interviews consist mainly of accepted and rejected statements in the proposed hypothesis as well as the user persona of a mother of preterm neonate.

The participant mothers of preterm neonates have been in the age group of 18-32 years. Most of these mothers are graduates from semi-urban areas around Pune, India. Most of them have been married when they were between 18 to 25 years and are housewives/homemakers. Most of the mothers prefer nonvegetarian diet and visit more frequently to pediatrician than a gynecologist. Most neonates are their first child and spontaneous preterm infants. These mothers are always eager to meet their neonates, especially for breastfeeding, but are not able to meet the neonates frequently; especially during nights. They do not use any mobile healthcare app. They do not join any user group related to neonate/pregnancy care on social media, but read related blogs. These mothers communicate with a physician in person and also, take appointments in person or over a phone. They do not use YouTube videos for information about neonate/ pregnancy care. Thus, the proposed mother's persona presents the details about demographic information, sources of information for mothers, their preferences, related facts, challenges and issues in the work environment of NICUs.

\section{Conclusion and Future Work}

In this paper, a user persona for Mother of Preterm Neonate has been proposed. This user persona has provided interesting insights into demographic information, sources of information for mothers, their preferences, related facts, challenges and issues in the work environment of NICUs. It will help application developers and usability professionals in the design of related healthcare applications. In the future, an Android app will be developed for the mothers of preterm neonates with due consideration to their user persona derived during this research work. Furthermore, how the app fits into the work of the healthcare staff in neonatal care, can also be addressed.

\section{Acknowledgement}

We thank India Alliance and The African Academy of Sciences (AAS) for providing Africa-India Mobility Fund (AIMF) Grant and supporting ICT research work related with Mothers of Preterm Infants.

\section{References}

1. Bouwstra, S., Chen, W., Feijs, L., Oetomo, S., Linden, W., Iisselsteijn, W.: Designing the Parents-to-Infant Bonding Experience in the NICU. First 
International Conference on Global Health Challenges, Oct. 2012, Venice, Italy, pp. 7-13. (2012)

2. Crude Birth Rate Statistics by World Data Atlas. https://knoema.com /atlas/India/topics/Demographics/Population-forecast/Crude-birth-rate. Accessed on $10^{\text {th }}$ April, 2019

3. Effective Population Growth. http://worldpopulationreview.com/countries /india-population/. Accessed on $23^{\text {rd }}$ April, 2019

4. Female Population in India. http://www.indiaonlinepages.com/population /india-current-population.html. Accessed on $24^{\text {th }}$ April, 2019

5. Goldberg, L., Lide, B., Lowry, S., Massett, H., O’Connell, T., Preece, J., Quesenbery, W., Shneiderman, B.: Usability and Accessibility in Consumer Health Informatics. American Journal of Preventive Medicine, 40(5), pp. S187-S197. (2011)

6. Heidari, H., Hasanpour, M., Fooladi, M.: The Iranian Parents of Premature Infants in NICU Experience Stigma of Shame. MED ARH, 66(1), pp. 35-40. (2012)

7. Mburu, W., Wardle, C., Joolay, Y., Densmore, M.: Co-designing with Mother and Neonatal Unit Staff: Use of Technology to Support Mothers of Preterm Infants. The $2^{\text {nd }}$ African Conference for Human-Computer Interaction - AfriCHI'18, Windhoek, Namibia. (2018)

8. Neonatal Intensive Care Unit (Wikipedia). https://en.wikipedia.org/wiki /Neonatal_intensive_care_unit. Accessed on 26 $6^{\text {th }}$ April, 2019

9. Neonatal Intensive Care Unit. https://www.stanfordchildrens.org/en/topic /default?id=the-neonatal-intensive-care-unit-nicu-90-P02389. Accessed on 22 $2^{\text {nd }}$ April, 2019

10. Nielsen, L.: 10 Steps to Personas. http://personas.dk/?pageid=196. Accessed on $26^{\text {th }}$ April, 2019.

11. Nielsen, L.: Personas. User Focused Design. Springer. (2019)

12. Patil, A., Bhutkar, G., Pendse, M., Tawade, A., Bodke, A., Shaha, S., Deshmukh, S.: Prototype Design of Android App for Mother of Preterm Infant. 5th IFIP WG 13.6 Working Conference, HWID 2018, Espoo, Finland. (2018)

13. van Velsen, L., van Gemert-Pijnen, L., Nijland, N., Beaujean, D., van Steenbergene J.: Personas: The Linking Pin in Holistic Design for eHealth. The Fourth International Conference on eHealth, Telemedicine, and Social Medicine - eTELEMED 2012, Valencia, Spain. (2012)

14. Vicini, S., Gariglio, A., Alberti, F., Oleari, E., Sanna, A.: Enhancing Personas for Well-Being e-Services and Product Service Systems. Design, User Experience, and Usability: Design Thinking and Methods - 5th International Conference, HCI International 2016, Springer Verlag, pp. 365-376. (2016)

15. Villalba, E., Peinado I., Arredondo, M.: User Interaction Design for a Wearable and IT-based Heart Failure System. 12th International Conference on Human-Computer Interaction, Beijing, China, pp. 1230-1239. (2007)

16. World Human Population. https://www.worldometers.info/world-population /india-population/. Accessed on $10^{\text {th }}$ April, 2019 